\title{
Hyperfine-Structure-Induced Depolarization of Impulsively Aligned I-2 Molecules
}

Thomas, Esben Folger; Sondergaard, Anders A.; Shepperson, Benjamin; Henriksen, Niels Engholm; Stapelfeldt, Henrik

Published in:

Physical Review Letters

Link to article, DOI:

10.1103/PhysRevLett.120.163202

Publication date:

2018

Document Version

Publisher's PDF, also known as Version of record

Link back to DTU Orbit

Citation (APA):

Thomas, E. F., Sondergaard, A. A., Shepperson, B., Henriksen, N. E., \& Stapelfeldt, H. (2018). HyperfineStructure-Induced Depolarization of Impulsively Aligned I-2 Molecules. Physical Review Letters, 120(16), [163202]. https://doi.org/10.1103/PhysRevLett.120.163202

\section{General rights}

Copyright and moral rights for the publications made accessible in the public portal are retained by the authors and/or other copyright owners and it is a condition of accessing publications that users recognise and abide by the legal requirements associated with these rights.

- Users may download and print one copy of any publication from the public portal for the purpose of private study or research.

- You may not further distribute the material or use it for any profit-making activity or commercial gain

- You may freely distribute the URL identifying the publication in the public portal 


\title{
Hyperfine-Structure-Induced Depolarization of Impulsively Aligned $\mathbf{I}_{2}$ Molecules
}

\author{
Esben F. Thomas, ${ }^{1}$ Anders A. Søndergaard, ${ }^{2}$ Benjamin Shepperson, ${ }^{2}$ Niels E. Henriksen, ${ }^{1}$ and Henrik Stapelfeldt ${ }^{2}$ \\ ${ }^{1}$ Department of Chemistry, Technical University of Denmark, Building 206, DK-2800 Kongens Lyngby, Denmark \\ ${ }^{2}$ Department of Chemistry, Aarhus University, Langelandsgade 140, DK-8000 Aarhus C, Denmark
}

(Received 30 January 2018; published 19 April 2018)

\begin{abstract}
A moderately intense 450 fs laser pulse is used to create rotational wave packets in gas phase $\mathrm{I}_{2}$ molecules. The ensuing time-dependent alignment, measured by Coulomb explosion imaging with a delayed probe pulse, exhibits the characteristic revival structures expected for rotational wave packets but also a complex nonperiodic substructure and decreasing mean alignment not observed before. A quantum mechanical model attributes the phenomena to coupling between the rotational angular momenta and the nuclear spins through the electric quadrupole interaction. The calculated alignment trace agrees very well with the experimental results.
\end{abstract}

DOI: 10.1103/PhysRevLett.120.163202

The alignment of isolated molecules, i.e., confinement of their internal axes to directions fixed in space, by moderately intense laser pulses is considered a well-understood process resulting from the polarizability interaction [1-4]. In the impulsive limit, where a laser pulse much shorter than the molecular rotational period is used, each molecule is left in a superposition of rotational eigenstates. For the widely studied case of linear molecules and a linearly polarized femtosecond alignment pulse, this wave packet formation causes the molecules to align shortly after the laser pulse and in periodically occurring narrow time windows, termed revivals [5-9]. In the rigid rotor approximation, the revival pattern repeats itself [10], unless the rotational coherence is distorted by, e.g., a dissipative environment [11-16].

Decades of frequency-resolved high-resolution spectroscopy $[17,18]$ and time-dependent depolarization experiments on molecules prepared in single rotational states (see Refs. [19-31] for previous examples) have, however, established that a rigid rotor model is insufficient and that a precise description of rotational spectra must include the coupling between rotational angular momentum and electronic or nuclear spin. It is, therefore, surprising that the influence of such effects, notably the hyperfine coupling between the electric quadrupole moment of the nuclei and the electric field of the electrons, has never been addressed in femtosecond-laser-induced molecular alignment studies. In the current work we measured the time-dependent degree of alignment, induced by a $450 \mathrm{fs}$ pulse, for a sample of $\mathrm{I}_{2}$ molecules covering the first seven rotational revivals. By contrast to the aforementioned depolarization studies, which do not involve coherent superpositions of rotational states, our experiment probes the impact of hyperfine coupling on the revival structures.

Using a quantum mechanical model in conjunction with the experimental results, we find that the hyperfine coupling affects the revival structures in qualitatively different ways compared to the well-understood impact on the "permanent" alignment of a molecule prepared in a single rotational state. Notably, the effect on the permanent alignment is known to be negligible in the limit where the rotational angular momentum is much larger than the angular momentum of the total nuclear spin [29]. By contrast, we find that the hyperfine coupling will always significantly perturb the revival structures over time.

The experimental setup and methods were described previously [32], so only a few details are pointed out here. A pulsed molecular beam, formed by expanding $\sim 1$ mbar iodine gas in 80 bar of He gas into a vacuum, enters a velocity map imaging spectrometer, where it is crossed at $90^{\circ}$ by two pulsed collinear laser beams. The first pulse (kick pulse, $\lambda=800 \mathrm{~nm}, \tau_{\text {FWHM }}=450 \mathrm{fs}, I_{0}=1.1 \times 10^{12} \mathrm{~W} / \mathrm{cm}^{2}$ ) creates rotational wave packets in the $\mathrm{I}_{2}$ molecules. The second pulse (probe pulse, $800 \mathrm{~nm}, 35 \mathrm{fs}, 4.3 \times 10^{14} \mathrm{~W} / \mathrm{cm}^{2}$ ) Coulomb explodes the molecules. This leads to $\mathrm{I}^{+}$ion fragments with recoil directions given by the angular distribution of the molecular axes at the instant of the probe pulse. The emission directions of the $\mathrm{I}^{+}$ions are recorded with a 2D imaging detector at different kick-probe delays, which allows us to determine the time-dependent degree of alignment $\left\langle\cos ^{2} \theta_{2 \mathrm{D}}\right\rangle, \theta_{2 \mathrm{D}}$ being the angle between the alignment pulse polarization and the projection of an $\mathrm{I}^{+}$ion velocity vector on the detector [33].

The time dependence of $\left\langle\cos ^{2} \theta_{2 \mathrm{D}}\right\rangle$ determined experimentally is shown in black in Fig. 1. The alignment trace is dominated by the pronounced half and full revivals, but their amplitude decreases with the revival order and their structure is changing. These observations are not caused by experimental factors such as collisions or limited temporal detection windows (see Supplemental Material [34], which includes Refs. [35-42]). For comparison, $\left\langle\cos ^{2} \theta_{2 \mathrm{D}}\right\rangle$ calculated by solving the time-dependent Schrödinger equation (TDSE) for a rigid rotor is also shown. It is clear that the 


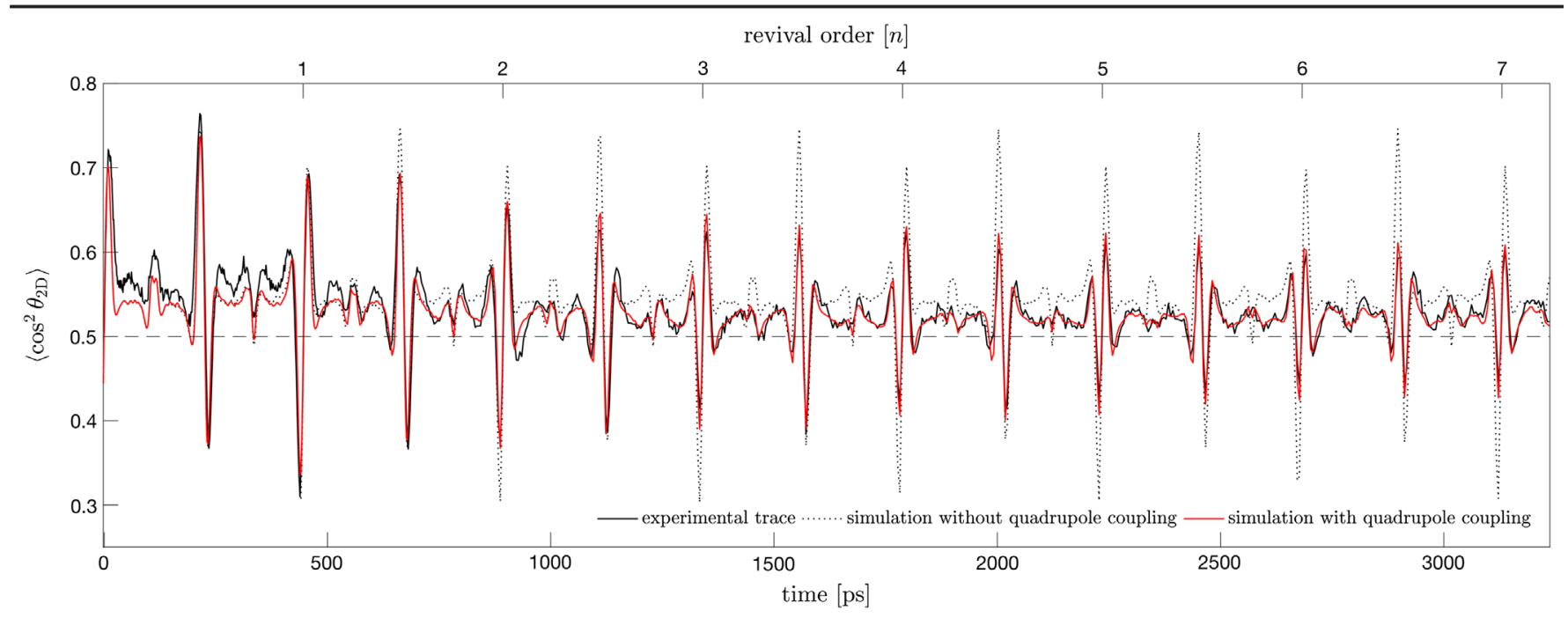

FIG. 1. Experimental results (black line) and the model calculations (red line) calculated at $T=0.8 \mathrm{~K}$. An alignment trace calculated without quadrupole coupling is also shown (dotted black line).

decreasing amplitude and changing structure of the revivals observed experimentally are at odds with the calculations. In particular, significant experimental deviations from the calculated results are evident in the higher-order fractional revivals; for instance, at the $6+(3 / 4)$ revival the experimental and calculated peaks point in opposite directions.

We now show that the numerical results match the experimental findings to a high degree of accuracy when hyperfine coupling is included in the theoretical model. For $\mathrm{I}_{2}$ molecules, this mainly stems from the coupling between the electric quadrupole moment of the atomic nuclei and the gradient of the electric field created by the electrons. The coupling between the magnetic dipole moment of the nuclei and the $B$ field from the electrons is much weaker and not included in our model [43]. The total nuclear spin $\mathbf{I}$ is the sum of the spins of the two atomic nuclei: $\mathbf{I}=\mathbf{I}_{1}+\mathbf{I}_{2}$. As a result, there are 36 nuclear spin isomers $\left|I M_{I}\right\rangle$ with $0 \leq I \leq$ 5 and $-I \leq M_{I} \leq I$, since the nuclear spin of ${ }^{127} \mathrm{I}$ is $5 / 2$. We assume that the 36 nuclear spin isomers are initially equally abundant [44]. The rotational wave packet created by the alignment pulse from an initial rotational eigenstate $\left|J_{i} M_{i}\right\rangle$ is denoted $\sum_{J} a_{J}\left|J M_{i}\right\rangle\left(M_{i}\right.$ is not changed due to the linear polarization of the alignment pulse). The symmetry requirements of the total molecular wave function entail that the parity of the $I$ and $J$ states must be the same in a given molecule [44]. Consequently, there are 15 (21) para (ortho) [even (odd) $I$ and $J$ ] spin isomers. The coupled spin isomerrotational wave packet is described as

$$
\left|I M_{I}\right\rangle \otimes \sum_{J} a_{J}\left|J M_{i}\right\rangle=\sum_{J} a_{J} \sum_{F} C_{M_{I} M_{i} M_{F}}^{I J F}\left|I J F M_{F}\right\rangle
$$

where $F$ is the total angular momentum, $\mathbf{F}=\mathbf{I}+\mathbf{J}$, $M_{F}=M_{I}+M_{i}$, and $C_{M_{I} M_{i} M_{F}}^{I J F}$ are the Clebsch-Gordan coefficients.
In preparation for solving the TDSE, we construct a square matrix $\mathbf{H}$ in the $\left|I J F M_{F}\right\rangle$ basis, with elements given by [45]

$$
\begin{aligned}
H_{a, b}= & \left\langle I^{a} J^{a} F^{a} M_{F}^{a}\left|\mathcal{H}_{B}+\mathcal{H}_{Q}\right| I^{b} J^{b} F^{b} M_{F}^{b}\right\rangle \\
= & \delta_{J^{b} J^{a}} B_{0} J^{a}\left(J^{a}+1\right) \\
& -(e q Q) \delta_{F^{b} F^{a}} \delta_{M_{F}^{b} M_{F}^{a}}\left[(-1)^{I^{a}}+(-1)^{I^{b}}\right](-1)^{F^{a}+I^{a}} \\
& \times\left[\frac{21}{20}\left(2 I^{b}+1\right)\left(2 I^{a}+1\right)\left(2 J^{b}+1\right)\left(2 J^{a}+1\right)\right]^{1 / 2} \\
& \times\left(\begin{array}{ccc}
J^{b} & 2 & J^{a} \\
0 & 0 & 0
\end{array}\right)\left\{\begin{array}{ccc}
F^{a} & I^{a} & J^{a} \\
2 & J^{b} & I^{b}
\end{array}\right\}\left\{\begin{array}{ccc}
\frac{5}{2} & I^{a} & \frac{5}{2} \\
I^{b} & \frac{5}{2} & 2
\end{array}\right\}
\end{aligned}
$$

$\mathcal{H}_{B}$ describes the rigid rotor Hamiltonian, where $B_{0}=$ $1.11863 \mathrm{GHz}$ [35] is the molecular rotational constant of $\mathrm{I}_{2}$ in the vibrational ground state (centrifugal distortion was found to be negligible [34]), and $\mathcal{H}_{Q}$ is the electric quadrupole interaction component of the hyperfine structure Hamiltonian for a diatomic molecule, where eqQ= $-2.45258 \mathrm{GHz}$ [43] is the quadrupole coupling constant.

$\mathcal{H}_{Q}$ introduces shifts in the diagonal elements of $\mathbf{H}$ and off-diagonal couplings when $\Delta I= \pm 2$ and/or $\Delta J= \pm 2$. Generally, $\mathbf{H}$ must incorporate all initial states occupied at $t=0$ [i.e., those given by the right side of Eq. (1)] as well as states that may become occupied over time as a result of the off-diagonal couplings. Specifically, it was found that any states that can be reached via inter- $I$ coupling must be included to faithfully reproduce the experimental alignment trace; however, inter- $J$ couplings were found to have a negligible impact on $\left\langle\cos ^{2} \theta_{2 \mathrm{D}}\right\rangle$ (attributed to the relatively large energy differences between various $J$ states). As such, the $\left|I J F M_{F}\right\rangle$ states incorporated in $\mathbf{H}$ need only contain $J$ values that were already present in $\sum_{J} a_{J}\left|J M_{i}\right\rangle$. 


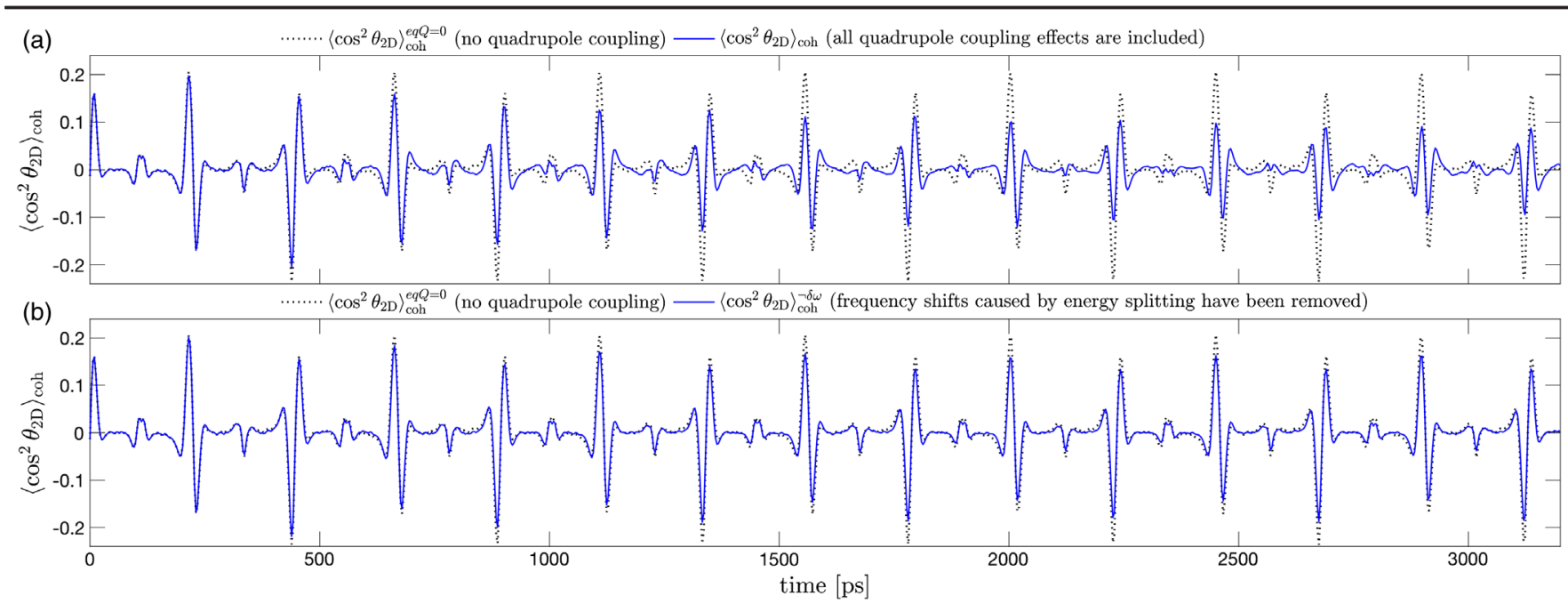

FIG. 2. In (a), the sum of $J \neq J^{\prime}$ matrix elements comprising the theoretical quadrupole coupled alignment trace $\left\langle\cos ^{2} \theta_{2 \mathrm{D}}\right\rangle_{\operatorname{coh}}$ is shown in blue. The blue trace in (b) shows $\left\langle\cos ^{2} \theta_{2 \mathrm{D}}\right\rangle_{\mathrm{coh}} \delta \omega$, where the frequency and phase shifts caused by the hyperfine energy splitting have been suppressed. Both (a) and (b) are superposed with equivalent traces calculated without quadrupole coupling (dotted black lines).

The TDSE is solved by expanding the full wave function $\Psi$ onto the coupled basis functions, i.e., $\Psi(t)=$ $\sum_{k=1}^{N} c_{k}(t)\left|I^{k} J^{k} F^{k} M_{F}^{k}\right\rangle$, where $N$ is the order of $\mathbf{H}$ and $\Psi(0)$ is given by Eq. (1). The $c_{k}(t)$ coefficients are found by diagonalizing $\mathbf{H}$ to solve the resulting system of coupled linear differential equations. $\Psi(t)$ is then transformed back into the uncoupled representation to calculate the alignment trace, i.e.,

$$
\Psi(t)=\sum_{k=1}^{N} c_{k}(t) \sum_{M_{I}, M_{J}} C_{M_{F}^{k} M_{I} M_{J}}^{F^{k} J^{k}}\left|I^{k} M_{I}\right\rangle \otimes\left|J^{k} M_{J}\right\rangle .
$$

The efficient calculation of $\left\langle\cos ^{2} \theta_{2 \mathrm{D}}\right\rangle$ is achieved by expanding $\cos ^{2} \theta_{2 \mathrm{D}}$ onto a basis of Legendre polynomials as described in Ref. [33] and noting the orthonormality of the $\left|I M_{I}\right\rangle$ states in Eq. (3).

The alignment trace of any initial $\sum_{J} a_{J}\left|J M_{i}\right\rangle$ superposition is the equally weighted, incoherent sum of traces generated by coupling to all nuclear spin isomers that symmetry requirements will permit. The complete alignment trace is the weighted incoherent sum of traces from all the different initial $\sum_{J} a_{J}\left|J M_{i}\right\rangle$ superpositions that exist because of thermal and focal volume averaging, as per the methodology outlined in Refs. [46,47].

The simulated alignment trace with the effects of quadrupole coupling included is shown in red in Fig. 1. Based on previous work in our group, we estimate that the molecules are initially in thermal (Boltzmann) equilibrium at $0.8 \mathrm{~K}$ [32]. The minor discrepancy between the theoretical and experimental traces from 0 to $600 \mathrm{ps}$ can essentially be eliminated by fitting the temperature [34]; however, this results in a fitted temperature of $\sim 0.4 \mathrm{~K}$, which we believe is unrealistically low.

Let $\sum_{J, J^{\prime}}\left\langle J M_{J}\left|\cos ^{2} \theta_{2 \mathrm{D}}\right| J^{\prime} M_{J}\right\rangle$ represent the sum of matrix elements that generates the theoretical alignment trace, where we omit the nuclear spin states and timedependent coefficients in Eq. (3) for clarity. The $J \neq J^{\prime}$ terms are sinusoidal functions oscillating at frequencies proportional to the energy difference between the $J$ and $J^{\prime}$ states. These terms represent the coherence of the wave packet and are responsible for the revivals. In analogy with previous work [11], we refer to their sum as $\left\langle\cos ^{2} \theta_{2 \mathrm{D}}\right\rangle_{\mathrm{coh}}$. Conversely, the terms where $J=J^{\prime}$ represent the population of the rotational states and characterize the permanent alignment. Their sum is denoted $\left\langle\cos ^{2} \theta_{2 \mathrm{D}}\right\rangle_{\text {perm }}$ [11]. Note that the mean alignment of the trace is well characterized by $\left\langle\cos ^{2} \theta_{2 \mathrm{D}}\right\rangle_{\text {perm }}$, as this term provides the baseline value around which $\left\langle\cos ^{2} \theta_{2 \mathrm{D}}\right\rangle_{\text {coh }}$ oscillates.

A visual inspection of Fig. 1 indicates that the mean alignment is slightly decreasing from 0 to $1000 \mathrm{ps}$. This behavior is attributed to the well-understood fact that quadrupole coupling leads to changes in the $M_{J}$ projection of a single $J$ state due to the angular "precession" of the coupled $\mathbf{I}$ and $\mathbf{J}$ vectors around $\mathbf{F}$ (see, e.g., Fig. 1 in Ref. [30]) and changes in the relative orientation of the individual nuclear spin vectors (which we denote "spin flipping"). Previous experiments on hyperfine-induced depolarization of single rotational states have shown that this effect (hereafter referred to as "precession-type depolarization") leads to a general time-dependent decrease in the molecular alignment $[19,20,22-31]$. Figure 2(a) shows $\left\langle\cos ^{2} \theta_{2 \mathrm{D}}\right\rangle_{\text {coh }}$ superposed with the sum of $J \neq J^{\prime}$ trace elements calculated without quadrupole coupling (denoted $\left\langle\cos ^{2} \theta_{2 \mathrm{D}}\right\rangle_{\operatorname{coh}^{e q} Q=0}$ ) for comparison. It is seen that the quadrupole coupling also strongly affects the revival structures.

To understand the cause of the amplitude loss and substructure modification in $\left\langle\cos ^{2} \theta_{2 \mathrm{D}}\right\rangle_{\text {coh }}$, the effects of precession-type depolarization were artificially suppressed in the model by changing all $M_{J}$ 's in Eq. (3) to the $M_{i}$ from 


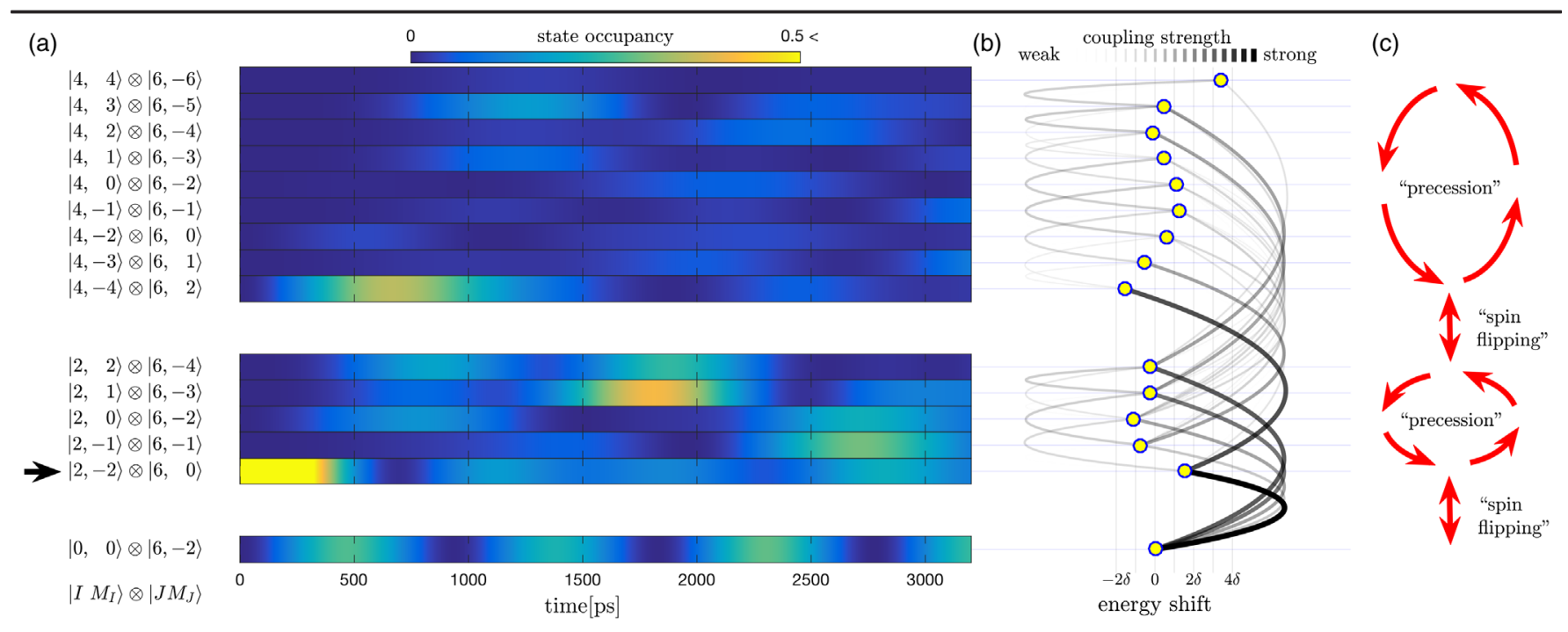

FIG. 3. (a) Occupancy of initial state $|2,-2\rangle \otimes|6,0\rangle$ and the $\left|I M_{I}\right\rangle \otimes\left|J M_{J}\right\rangle$ states it couples to evolving over the timescale of the experiment. (b) Sketch of the state energy splittings and relative coupling strengths ( $\delta=57 \mathrm{MHz}$ ). (c) Schematic classical interpretation of the system dynamics.

the initial $\sum_{J} a_{J}\left|J M_{i}\right\rangle$ superposition when calculating the $\left\langle J M_{J}\left|\cos ^{2} \theta_{2 \mathrm{D}}\right| J^{\prime} M_{J}\right\rangle$ elements (while treating everything else as if the "actual" $M_{J}$ 's are still in place). Surprisingly, this has very little effect on the shape of $\left\langle\cos ^{2} \theta_{2 \mathrm{D}}\right\rangle_{\mathrm{coh}}$, indicating that some previously unexplored mechanisms associated with the quadrupole coupling are causing the modulations in the signal.

It is informative to show the quadrupole coupled dynamics of a molecule starting in a single spin isomer/ $J$ state combination. In Fig. 3(a), the time evolution of initial state $|2,-2\rangle \otimes|6,0\rangle$ is shown projected onto the $\left|I M_{I}\right\rangle \otimes\left|J M_{J}\right\rangle$ basis, where the (negligible) effect of inter- $J$ coupling has been suppressed for clarity. The example in Fig. 3 illustrates how the quadrupole coupling will cause each $\left|J M_{i}\right\rangle$ from the initial $\sum_{J} a_{J}\left|J M_{i}\right\rangle$ superposition to spread out across a " $J$ manifold" of coupled states. Also, Fig. 3 shows how all states in a given $J$ manifold will have different $\left|I M_{I}\right\rangle$. Therefore, orthonormality of the $\left|I M_{I}\right\rangle$ spin states implies that each state in the $J$ manifold will combine with at most one state in the $J^{\prime}$ manifold to yield a nonzero contribution to the alignment trace.

Given two or more superposed $J$ states coupled to the same spin isomer at $t=0$, the energy splitting, coupling strength, and number of states associated with each $J$ manifold partially depend on $J$ [attributable, e.g., to the appearance of $J^{a, b}$ in Eq. (2)]. Dissimilarities in the energy splitting between different $J$ manifolds introduce multiple frequency shifts into the components of $\left\langle\cos ^{2} \theta_{2 \mathrm{D}}\right\rangle_{\mathrm{coh}}$. The beating caused by the introduction of these new frequencies modulates the alignment trace. We investigated the nature of this frequency beating by artificially suppressing its effect in the model. This was done by eliminating the quadrupole-coupling-induced frequency and phase shifts in the complex arguments of the coefficients governing the time evolution of all $\left|I M_{I}\right\rangle \otimes\left|J M_{J}\right\rangle$ states across all $J$ manifolds. In this way, we calculate a modified trace $\left\langle\cos ^{2} \theta_{2 \mathrm{D}}\right\rangle_{\text {coh }}^{\neg \delta \omega}$, where $\neg \delta \omega$ indicates that all frequency shifts introduced by the energy splitting have been removed while leaving the $J$-manifold population dynamics unchanged. A plot of $\left\langle\cos ^{2} \theta_{2 \mathrm{D}}\right\rangle_{\text {coh }}^{\neg \delta \omega}$ is shown in Fig. 2(b). A comparison of the $\left\langle\cos ^{2} \theta_{2 \mathrm{D}}\right\rangle_{\mathrm{coh}}$ and $\left\langle\cos ^{2} \theta_{2 \mathrm{D}}\right\rangle_{\text {coh }}^{\neg \delta \omega}$ traces shown in Fig. 2 reveals that the frequency beating plays a significant, but not singular, role in attenuating the peak amplitudes. It is also remarkable that the higher-order fractional revivals in $\left\langle\cos ^{2} \theta_{2 \mathrm{D}}\right\rangle_{\text {coh }}^{\neg \delta \omega}$ do not exhibit the deviations and sign changes that are present in $\left\langle\cos ^{2} \theta_{2 \mathrm{D}}\right\rangle_{\mathrm{coh}}$. This demonstrates that the complex nonperiodic substructures observed in the experimental trace can be solely attributed to the new frequencies introduced into $\left\langle\cos ^{2} \theta_{2 \mathrm{D}}\right\rangle_{\text {coh }}$ by the hyperfine coupling.

Note that the peak amplitudes in $\left\langle\cos ^{2} \theta_{2 \mathrm{D}}\right\rangle_{\mathrm{coh}}{ }_{\mathrm{coh}}$ still decrease compared to $\left\langle\cos ^{2} \theta_{2 \mathrm{D}}\right\rangle_{\mathrm{coh}}^{e q Q=0}$. This is because, as stated earlier, the state population distributions of manifolds with different $J$ will become increasingly dissimilar over time. These asynchronous distributional dynamics cause a net loss of amplitude due to the bijective or injective (one to at most one) way of combining different sets of states associated with different $J$ manifolds when calculating nonzero contributions to the trace. Experimentally, some of the observed peak attenuation may, in principle, be caused by $\mathrm{I}_{2}$ molecules in vibrationally excited states. Our analysis shows, however, that the potential impact is minor [34].

It has been remarked that precession-type depolarization in single $J$ states is most significant when the coupled $\mathbf{I}$ and $\mathbf{J}$ vectors have similar magnitudes [29]. Conversely, our analysis suggests that the observed modulations in the revival structures of $\left\langle\cos ^{2} \theta_{2 \mathrm{D}}\right\rangle_{\text {coh }}$ are not directly contingent on the magnitude of $\mathbf{I}$ or $\mathbf{J}$. Therefore, we investigate 


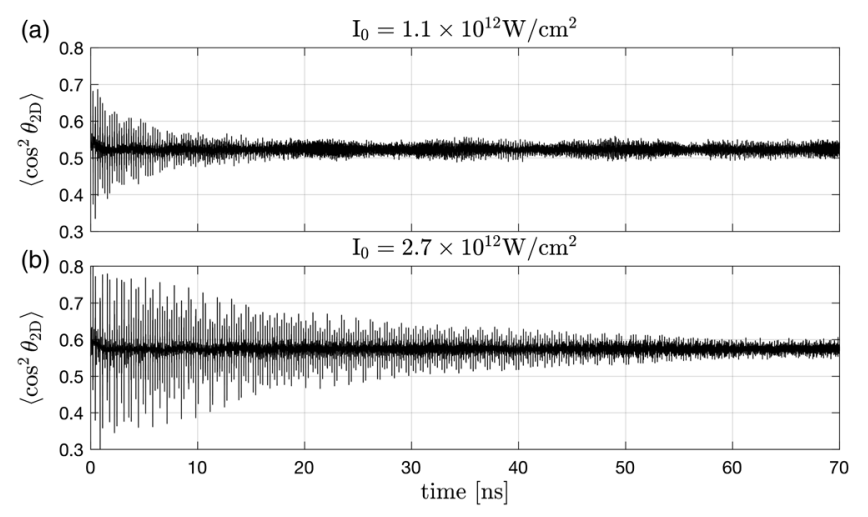

FIG. 4. Quadrupole-coupled alignment traces simulated to $70 \mathrm{~ns}$, with alignment pulse intensities set to (a) the experimental and (b) $2.5 \times$ the experimental value.

what happens if rotational wave packets containing larger $J$ are created. To this end, we simulated the effects of quadrupole coupling in $\mathrm{I}_{2}$ molecules aligned with pulses up to $9 \times$ more intense than used in the current experiment.

Increasing the pulse intensity leads to initial revival peaks with larger amplitudes, as well as a higher level of mean alignment. The early decrease in the mean alignment observed in the experiment becomes less pronounced at higher intensities, and for all intensities the mean alignment is nearly constant when $t>1 \mathrm{~ns}$. Additionally, it was found that for all intensities the revival structures always decay into what resembles low-amplitude unstructured "noise"; however, this decay takes longer for more intense pulses, as illustrated in Fig. 4 [34]. These observations agree qualitatively with our expectations; i.e., the classical model of precession predicts that $\left\langle\cos ^{2} \theta_{2 \mathrm{D}}\right\rangle_{\text {perm }}$ will change less for wave packets with large $J$, whereas the scrambling or attenuating effects of the frequency beating and asynchronous dynamics will accumulate over time and eventually dominate the $\left\langle\cos ^{2} \theta_{2 \mathrm{D}}\right\rangle_{\mathrm{coh}}$ component of the trace regardless of the magnitude of the $J$ values present in the wave packet.

In closing, we note that the alignment trace of any molecule containing heavy atoms (e.g., I or Br) with large quadrupole coupling constants is expected to show similar deviations from the rigid rotor approximation when excited into a coherent superposition of rotational eigenstates.

H. S. acknowledges support from the European Research Council-AdG (Project No. 320459, DropletControl).

[1] H. Stapelfeldt and T. Seideman, Colloquium: Aligning molecules with strong laser pulses, Rev. Mod. Phys. 75, 543 (2003).

[2] T. Seideman and E. Hamilton, Nonadiabatic alignment by intense pulses. Concepts, theory, and directions, Adv. At. Mol. Opt. Phys. 52, 289 (2005).

[3] Y. Ohshima and H. Hasegawa, Coherent rotational excitation by intense nonresonant laser fields, Int. Rev. Phys. Chem. 29, 619 (2010).
[4] S. Fleischer, Y. Khodorkovsky, E. Gershnabel, Y. Prior, and I. Sh. Averbukh, Molecular alignment induced by ultrashort laser pulses and its impact on molecular motion, Isr. J. Chem. 52, 414 (2012).

[5] T. Seideman, Revival Structure of Aligned Rotational Wave Packets, Phys. Rev. Lett. 83, 4971 (1999).

[6] F. Rosca-Pruna and M. J. J. Vrakking, Experimental Observation of Revival Structures in Picosecond Laser-Induced Alignment of $I_{2}$, Phys. Rev. Lett. 87, 153902 (2001).

[7] M. Machholm and N. E. Henriksen, Field-Free Orientation of Molecules, Phys. Rev. Lett. 87, 193001 (2001).

[8] V. Renard, M. Renard, S. Guerin, Y. T. Pashayan, B. Lavorel, O. Faucher, and H. R. Jauslin, Postpulse Molecular Alignment Measured by a Weak Field Polarization Technique, Phys. Rev. Lett. 90, 153601 (2003).

[9] P. W. Dooley, I. V. Litvinyuk, Kevin F. Lee, D. M. Rayner, M. Spanner, D. M. Villeneuve, and P. B. Corkum, Direct imaging of rotational wave-packet dynamics of diatomic molecules, Phys. Rev. A 68, 023406 (2003).

[10] A. Przystawik, A. Kickermann, A. Al-Shemmary, S. Düsterer, A. M. Ellis, K. von Haeften, M. Harmand, S. Ramakrishna, H. Redlin, L. Schroedter, M. Schulz, T. Seideman, N. Stojanovic, J. Szekely, F. Tavella, S. Toleikis, and T. Laarmann, Generation of the simplest rotational wave packet in a diatomic molecule: Tracing a two-level superposition in the time domain, Phys. Rev. A 85, 052503 (2012).

[11] S. Ramakrishna and T. Seideman, Intense Laser Alignment in Dissipative Media as a Route to Solvent Dynamics, Phys. Rev. Lett. 95, 113001 (2005).

[12] T. Vieillard, F. Chaussard, D. Sugny, B. Lavorel, and O. Faucher, Field-free molecular alignment of $\mathrm{CO}_{2}$ mixtures in presence of collisional relaxation, J. Raman Spectrosc. 39, 694 (2008).

[13] N. Owschimikow, F. Knigsmann, J. Maurer, P. Giese, A. Ott, B. Schmidt, and N. Schwentner, Cross sections for rotational decoherence of perturbed nitrogen measured via decay of laser-induced alignment, J. Chem. Phys. 133, 044311 (2010).

[14] J. M. Hartmann and C. Boulet, Quantum and classical approaches for rotational relaxation and nonresonant laser alignment of linear molecules: A comparison for $\mathrm{CO}_{2}$ gas in the nonadiabatic regime, J. Chem. Phys. 136, 184302 (2012).

[15] D. Pentlehner, J. H. Nielsen, A. Slenczka, K. Mølmer, and H. Stapelfeldt, Impulsive Laser Induced Alignment of Molecules Dissolved in Helium Nanodroplets, Phys. Rev. Lett. 110, 093002 (2013).

[16] B. Shepperson, A. A. Søndergaard, L. Christiansen, J. Kaczmarczyk, R. E. Zillich, M. Lemeshko, and H. Stapelfeldt, Laser-Induced Rotation of Iodine Molecules in Helium Nanodroplets: Revivals and Breaking Free, Phys. Rev. Lett. 118, 203203 (2017).

[17] W. Gordy and R. L. Cook, Microwave Molecular Spectra, 3rd ed. (Wiley, New York, 1984).

[18] R. N. Zare, Angular Momentum (Wiley, New York, 1988).

[19] R. F. Code and N. F. Ramsey, Molecular-beam magnetic resonance studies of $\mathrm{HD}$ and $\mathrm{D}_{2}$, Phys. Rev. A 4, 1945 (1971).

[20] U. Fano and J. H. Macek, Impact excitation and polarization of the emitted light, Rev. Mod. Phys. 45, 553 (1973). 
[21] R. Altkorn, R. N. Zare, and C. H. Greene, Depolarization of optically prepared molecules by two randomly oriented spins, Mol. Phys. 55, 1 (1985).

[22] C. Yan and A. C. Kummel, Effect of hyperfine depolarization upon creation and detection of alignment in free-jet expansions via selective photodissociation, J. Chem. Phys. 98, 6869 (1993).

[23] S. F. Gough and A. Crowe, The effect of hyperfine depolarization on electron-photon angular correlation measurements in krypton, J. Phys. B 26, 2403 (1993).

[24] T. A. Cool and N. Hemmi, Hyperfine polarization quantum beats in cyanogen, J. Chem. Phys. 103, 3357 (1995).

[25] J. Zhang, C. W. Riehn, M. Dulligan, and C. Wittig, An experimental study of HF photodissociation: Spin-orbit branching ratio and infrared alignment, J. Chem. Phys. 104, 7027 (1996).

[26] E. R. Wouters, L. D. A. Siebbeles, K. L. Reid, B. Buijsse, and W. J. van der Zande, Observation of fine structure and hyperfine structure depolarization in the photofragment anisotropy in triplet $\mathrm{H}_{2}$, Chem. Phys. 218, 309 (1997).

[27] A. D. Rudert, J. Martin, W.-B. Gao, J. B. Halpern, and H. Zacharias, Collisional effects on angular momentum orientation in acetylene $\tilde{X}^{1} \Sigma_{g}^{+}\left(\nu_{2}^{\prime \prime}=1, j^{\prime \prime}\right)$. I. Preparation, detection and conservation in single collisions, J. Chem. Phys. 111, 9549 (1999).

[28] D. Sofikitis, L. Rubio-Lago, M. R. Martin, D. J. Ankeny Brown, N. C.-M. Bartlett, A. J. Alexander, R. N. Zare, and T. P. Rakitzis, Optical control of ground-state atomic orbital alignment: $\mathrm{Cl}\left({ }^{2} P_{3 / 2}\right)$ from $\mathrm{HCl}(\mathrm{v}=2, \mathrm{j}=1)$ photodissociation, J. Chem. Phys. 127, 144307 (2007).

[29] N. C.-M. Bartlett, D. J. Miller, R. N. Zare, A. J. Alexander, D. Sofikitis, and T. P. Rakitzis, Time-dependent depolarization of aligned HD molecules, Phys. Chem. Chem. Phys. 11, 142 (2009).

[30] N. C.-M. Bartlett, J. Jankunas, R. N. Zare, and J. A. Harrison, Time-dependent depolarization of aligned $\mathrm{D}_{2}$ caused by hyperfine coupling, Phys. Chem. Chem. Phys. 12, 15689 (2010).

[31] K. Grygoryeva, J. Rakovský, O. Votava, and M. Fárník, Imaging of rotational wave-function in photodissociation of rovibrationally excited $\mathrm{HCl}$ molecules, J. Chem. Phys. 147, 013901 (2017).

[32] B. Shepperson, A. S. Chatterley, A. A. Søndergaard, L. Christiansen, M. Lemeshko, and H. Stapelfeldt, Strongly aligned molecules inside helium droplets in the nearadiabatic regime, J. Chem. Phys. 147, 013946 (2017).
[33] A. A. Søndergaard, B. Shepperson, and H. Stapelfeldt, Nonadiabatic laser-induced alignment of molecules: Reconstructing $\left\langle\cos ^{2} \theta\right\rangle$ directly from $\left\langle\cos ^{2} \theta_{2 \mathrm{~d}}\right\rangle$ by Fourier analysis, J. Chem. Phys. 147, 013905 (2017).

[34] See Supplemental Material at http://link.aps.org/ supplemental/10.1103/PhysRevLett.120.163202 for extended simulations and analyses.

[35] NIST Chemistry WebBook, NIST Standard Reference Database Number 69, edited by P. J. Linstrom and W. G. Mallard (National Institute of Standards and Technology, Gaithersburg, MD, 2017), http://webbook.nist.gov.

[36] R. Bacis, M. Broyer, S. Churassy, J. Vergès, and J. Vigué, eQq measurements in the $\mathrm{X}, 1 \mathrm{~g}, \mathrm{O}_{\mathrm{g}}^{+}$and $\mathrm{B}$ state of $\mathrm{I}_{2}$ : A test of the electronic molecular eigenfunctions, J. Chem. Phys. 73, 2641 (1980).

[37] D. M. Lubman, C. T. Rettner, and R. N. Zare, How isolated are molecules in a molecular beam?, J. Phys. Chem. 86, 1129 (1982).

[38] G. Maroulis, Accurate dipole polarizability for $\mathrm{Cl}_{2}\left(\mathrm{X}^{1} \Sigma_{\mathrm{g}}^{+}\right)$, Mol. Phys. 77, 1085 (1992).

[39] G. Maroulis, C. Makris, U. Hohm, and D. Goebel, Electrooptical properties and molecular polarization of iodine, $\mathrm{I}_{2}$, J. Phys. Chem. A 101, 953 (1997).

[40] U. Even, J. Jortner, D. Noy, N. Lavie, and C. CossartMagos, Cooling of large molecules below $1 \mathrm{~K}$ and $\mathrm{He}$ clusters formation, J. Chem. Phys. 112, 8068 (2000).

[41] F. Filsinger, J. Küpper, G. Meijer, L. Holmegaard, J. H. Nielsen, I. Nevo, J. L. Hansen, and H. Stapelfeldt, Quantumstate selection, alignment, and orientation of large molecules using static electric and laser fields, J. Chem. Phys. 131, 064309 (2009); https://aip.scitation.org/doi/full/10.1063/1 .3194287 .

[42] C.-C. Shu, E. F. Thomas, and N. E. Henriksen, Femtochemistry in the electronic ground state: Dynamic stark control of vibrational dynamics, Chem. Phys. Lett. 683, 234 (2017).

[43] A. Yokozeki and J. S. Muenter, Laser fluorescence state selected and detected molecular beam magnetic resonance in $\mathrm{I}_{2}$, J. Chem. Phys. 72, 3796 (1980).

[44] D. A. McQuarrie, Statistical Mechanics (Harper \& Row, New York, 1976).

[45] R. L. Cook and F. C. De Lucia, Application of the theory of irreducible tensor operators to molecular hyperfine structure, Am. J. Phys. 39, 1433 (1971).

[46] C. Z. Bisgaard, Ph.D. thesis, Aarhus University, 2006.

[47] A. A. Søndergaard, Ph.D. thesis, Aarhus University, 2016. 